\title{
Influence de la recombinaison sur la variabilité génétique. I. Étude expérimentale
}

\author{
N Robert ${ }^{1^{*}}$, J Kervella ${ }^{2}$, G Fouilloux ${ }^{3}$ \\ 1 INRA, station d'amélioration des plantes, domaine de Crouelle, F 63039 Clermont-Ferrand Cedex; \\ 2 INRA, station de recherches fruitières méditerranéennes, BP 91, F 84143 Montfavet Cedex; \\ ${ }^{3}$ CNRA, station d'amélioration des plantes, route de Saint-Cyr, $F 78000$ Versailles, France
}

(Reçu le 22 février 1991; accepté le 6 janvier 1993)

\begin{abstract}
Résumé - Afin d'étudier l'intérêt éventuel d'un gène modificateur des fréquences de recombinaison pour l'amélioration des plantes, une étude expérimentale a été entreprise chez le pétunia. L'effet d'un allèle élévateur des fréquences de recombinaison (allèle $\mathrm{Rm} 1$ ) sur la variabilité de caractères quantitatifs a été étudié. Pour cela, 2 groupes de 5 plantes différant par leur génotype au locus Rm1 ont été constitués. Quatre caractères morphologiques et un caractère phénologique ont été étudiés dans les familles issues des 5 plantes de chaque groupe par un plan circulaire de croisement, par 1 et par 2 générations d'autofécondation. Les variances inter- et intrafamille ont été comparées au sein de chaque groupe en fonction du type de descendance, et d'un groupe à l'autre pour chaque type de descendance. Pour un type de descendance donné, les variances inter- et intrafamilles ne diffèrent pas d'un groupe à l'autre. Elles évoluent en fonction du type de descendance, de façon similaire dans les 2 groupes. Les raisons de cette absence de différence entre groupes sont discutées.
\end{abstract}

recombinaison / variabilité génétique / variance / pétunia

Summary - Influence of recombination on genetic variability. I. An experimental study. In order to evaluate the possible interest for plant breeding of a gene modifying recombination rates, an experimental study was undertaken on petunia, as in this species an allele (allele $R \mathrm{~m} 1$ ) increases recombination rates. The effect of this allele $R m 1$ on genetic variability of quantitative traits was studied. A group of $5 \mathrm{Rm} 1 / \mathrm{rm} 1$ plants and a group of $5 \mathrm{rm} 1 / \mathrm{rm} 1$ plants were studied. Four morphological traits and 1 phenological trait were measured in the populations derived from the 5 plants of each group, either by generation of a circular mating design (SIB population), or by 1 (AF1 populations) or 2 generations (AF2 populations) of selfing. Within and between-family variances in these populations were compared within each group, and between groups for each kind of progeny. Although differences existed between SIB, AF1 and AF2 populations within each group, no significant difference was found between groups for each kind of progeny. Possible reasons for the lack of differences between groups are discussed.

recombination / genetic variability / variance / petunia

\section{INTRODUCTION}

Deux phénomènes coexistent à la méiose : la recombinaison interchromosomique ou ségrégation, et la recombinaison intrachromosomique entre loci liés, qui conduit à l'échange de segments homologues. Mather $(1941,1942)$ et Spiess et Allen (1961), travaillant sur drosophile, espèce où certaines souches ne présentent pas de recombinaison intrachromosmique, ont montré que la recombinaison crée des com- binaisons nouvelles de gènes et que la variation fournie par les recombinaisons intrachromosomiques est plus élevée que celle fournie par les ségrégations.

Dans l'espèce Petunia hybrida, certains segments chromosomiques, délimités par des gènes à effet qualitatif (gènes marqueurs), ne présentent pas ou peu de recombinaison (Cornu et al, 1990). L'étude des liaisons génétiques entre les gènes marqueurs identifiés chez cette espèce a permis de révéler la présence

\footnotetext{
* Correspondance et tirés à part
} 
d'un gène modificateur des taux de recombinaison (locus Rm1) dont l'allèle Rm1 élève généralement les taux de recombinaison (Mousset, 1985; Farcy et al, 1986). Dans des descendances d'effectif raisonnable (200 - 300 plantes) issues de test-cross, seules sont présentes les combinaisons parentales en l'absence de l'allèle $\mathrm{Rm} 1$ (plantes $\mathrm{rm} 1 / \mathrm{rm} 1$ ); en revanche, en présence de $\mathrm{Rm} 1$, des combinaisons géniques issues de recombinaison apparaissent avec une fréquence notable (Mousset, 1985; Cornu et al, 1989; Robert et al, 1991). Pour les caractères qualitatifs considérés, le gène Rm1 permet donc d'augmenter la variabilité génétique, définie comme la diversité des combinaisons géniques.

Qu'en est-il des caractères quantitatifs, définis comme des caractères contrôlés par des gènes nombreux à effet mineur ou polygènes (Mather, 1943) ? Un gène accroissant les fréquences de recombinaison (similaire à celui présent chez $P$ hybrida) présente-t-il un intérêt pour le sélectionneur? Pourrait-il permettre une gestion plus efficace de la variabilité génétique, conduisant à l'obtention plus rapide des combinaisons géniques les mieux adaptées ? La variance, classiquement utilisée pour mesurer la variabilité génétique d'une population, est-elle un outil satisfaisant pour traduire la variabilité des combinaisons de gènes et les remaniements qu'elles subissent après chaque méiose?

Cet article présente une étude expérimentale entreprise sur Petunia hybrida pour répondre à ces questions. Au cours de cette étude, les variances de caractères quantitatifs ont été calculées dans plusieurs populations possédant ou ne possédant pas l'allèle Rm1.

\section{ÉTUDE EXPÉRIMENTALE SUR PÉTUNIA}

\section{Matériels et méthodes}

\section{Matériel végétal}

Le protocole d'obtention du matériel de départ (plantes mères) est résumé dans la figure 1.

Les plantes mères sont issues du croisement (TLh1 $\times$ Drm $) \times(\operatorname{Drm} \times$ Sp 13), les 3 génotypes n'étant pas apparentés. TLh1 et Sp13 sont 2 lignées homozygotes (coefficient de consanguinité $F=1$ ), ne possédant pas la capacité d'augmenter les fréquences de recombinaison car elles sont homozygotes récessives rm1/rm1. Drm est une plante partiellement consanguine, issue d'un croisement complexe suivi de plusieurs autofécondations $(F=0,78)$, homozygote $\mathrm{Rm} 1$ / $\mathrm{Rm} 1$, induisant des augmentations de recombinaison dans ses descendances en croisement.

En se basant sur les taux de recombinaison dans 2 segments chromosomiques délimités par des gènes marqueurs : segment Hf1-Lg1 sur le chromosome I, segment An2-Rt sur le chromosome VI, 2 groupes de plantes ont été constitués, le groupe $A$ composé de plante $\mathrm{rm} 1 / \mathrm{rm} 1$, le groupe $B$ composé de plantes Rm1/Rm1 (fig 1). Cette méthode de détermination du génotype au locus Rm1 étant très lourde, les contraintes liées à ce protocole n'ont permis d'obtenir que 5 plantes par groupe.

Deux modes de reproduction ont été appliqués à ces plantes : le croisement et l'autofécondation (modalités présentées fig 2) :

- les 5 plantes de chaque groupe ont été intercroisées selon un plan circulaire. Le coefficient de consanguinité de ces familles, désignées par SIB, était de 0,36.

- les plantes mères de chaque groupe ont été autofécondées. Le coefficient de consanguinité de ces familles, désignées par AF1, était de 0,61. Dans chaque

croisement avec un testeur :

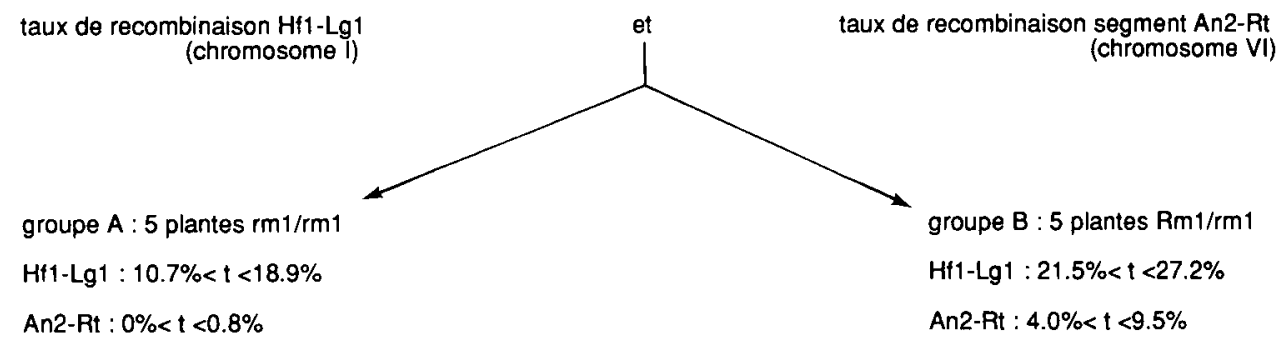

Fig 1. Obtention du matériel végétal : plantes mères. 


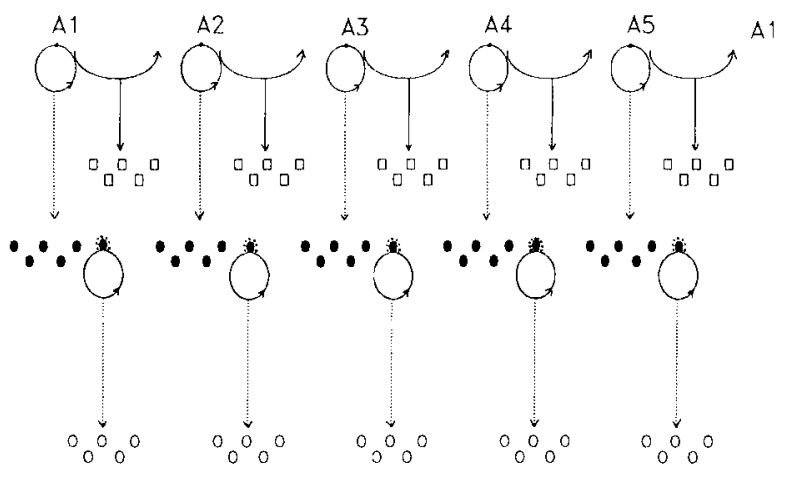

Fig 2. Gestion du matériel végétal : obtention des populations $\mathrm{SIB}, \mathrm{AF}_{1}, \mathrm{AF}_{2}$. Individu de la population $\mathrm{AF}$, observé $\bullet$; autofécondé: individu de la population AF2 observé $\mathrm{O}$; individu de la population SIB observé $\square$.

famille, une plante a été retenue et autofécondée. Les familles AF2 observées $(F=0,80)$ sont issues de ces plantes. Les plantes retenues dans le groupe $B$ devant être hétérozygotes $\mathrm{Rm} 1 / \mathrm{rm} 1$, le contrôle du génotype au locus Rmt a été nécessaire pour retenir ces plantes.

Les populations SIB, AF1 et AF2 des groupes A et $B$ ont été cultivés simultanément en serre, dans des multipots contenant chacun 16 plantes bordées. Pour des raisons pratiques, toutes les plantes cultivées dans un même multipot appartenaient à une même famille. Pour chaque famille, 4 multipots ont été constitués. Ils ont été placés selon un dispositif complètement randomisé.

De 56 à 64 plantes ont été observées par famille, l'effectif total des populations SIB, AF1 et AF2 variait de 300 à 317 .

\section{Caractères étudiés}

Cinq caractères quantitatifs ont été observés :

$P V$ : longueur de la période végétative (j),

$H T$ : hauteur de la tige du sol aux dernières feuilles sous la première fleur $(\mathrm{cm})$,

$D M$ : diamètre sous la dernière feuille alterne $(\mathrm{cm})$,

$L F$ : largeur de cette feuille $(\mathrm{cm})$,

$L C$ : longueur de la première corolle $(\mathrm{cm})$.

\section{Modèle statistique}

La variation phénotypique observée a été décomposée selon un modèle hiérarchique à effets aléatoires:

$$
x_{i j k}=\mu+F_{i}+M_{i j}+I_{i j k}
$$

$\mu$ : moyenne de la population de référence;

$F_{i}$ : effet moyen de la famille $i$, il dépend du génotype des plantes mères de la famille; $\sigma_{F}^{2}$ variance interfamilles,
$M_{i j}$ : effet de multipot, $\sigma^{2} M$ variance inter-multipots (macro-environnementale);

$l_{i j k}$ : effet individuel, $\sigma^{2}$, variance intrafamille. La variatíon entre les individus de la même famille, hormis l'effet multipot, a deux origines possibles : une origine génétique, le matériel étudié étant en ségrégation, et une origine micro-environnementale (intra-multipot). L'utilisation de génotypes témoins (lignées et hybrides $F_{1}$ ) ne nous a pas permis d'estimer la variance microenvironnementale : en effet, pour certains caractères, la variance intrafamille moyenne des lignées et des hybrides était supérieure à celle des populations en ségrégation (Robert, 1989). Nous avons donc comparé les variances intrafamille $\sigma^{2}$.

Les comparaisons des différentes variances des trois populations $\mathrm{SIB}, \mathrm{AF}_{1}, \mathrm{AF}_{2}$ d'un même groupe ont été effectuées à l'aide d'un test de Bartlett; les comparaisons entre groupes par un test de $F$. Les variances $\sigma_{F}^{2}$ étant des composantes de variance obtenues par soustraction de plusieurs carrés moyens, elle ne suivaient pas une distribution de $\chi^{2}$ et ne pouvaient pas être comparées par un test de Bartlett. Nous avons calculé les estimateurs des effets "famille": $F_{i}=\bar{x}_{i}$. $\bar{x} . . ., \bar{x}_{i}$. moyenne de la famille $i, \bar{x} .$. moyenne générale. Les carrés moyens de ces estimateurs ont été calculés puis comparés par un test de Bartlett.

\section{RÉSULTATS}

Une seule variance estimée est négative (composante $\sigma_{F}^{2}$ pour le caractère HT, population SIB du groupe B). Le modèle statistique choisi est considéré comme correct et cette variance est déclarée nulle (Searle, 1971).

Les variances des effets du macroenvironnement sont plus faibles que les variances intra- et interfamilles $\sigma^{2}$, et $\sigma_{F}^{2}$. La variance $\sigma_{F}^{2}$ est elle-même plus faible que $\sigma_{l}^{2} \mathrm{La}$ part environnementale de $\sigma^{2}$, semble importante, probablement plus que la part génétique, car les variances $\sigma^{2}$, ne sont guère plus élevées que celles obtenues pour des lignées ou des hybrides cultivés simultanément (Robert, 1989).

\section{Comparaison des 3 générations d'un même groupe}

\section{Variances intrafamille $\sigma^{2}$ (tableau I)}

Le nombre de degrés de liberté affecté à chaque variance étant élevé, les résultats des tests sont fiables. Dans chaque groupe, il existe des différences entre les variances intrafamilles des populations $\mathrm{SIB}, A \mathrm{~F}_{1}, A \mathrm{~F}_{2}$, excepté pour la longueur de la corolle (LC) dans le groupe $B$. 
Tableau I. Variances intrafamilles $\sigma_{1}^{2}$ : valeurs et comparaisons par un test de Bartlett pour les 3 populations.

\begin{tabular}{|c|c|c|c|c|}
\hline Variable concernée & $S I B$ & $A F_{1}$ & $A F_{2}$ & Probabilité \\
\hline \multicolumn{5}{|l|}{ Groupe A } \\
\hline$d l$ & 294 & 294 & 273 & \\
\hline PV & 11,4 & 12,9 & 6,6 & $0 *$ \\
\hline$H T$ & 11,84 & 12,22 & 9,50 & 7,9 NS \\
\hline$D M$ & $0,991 \cdot 10^{-3}$ & $0,651 \cdot 10^{-3}$ & $1,003.10^{-3}$ & $0,02^{*}$ \\
\hline$L F$ & 0,12 & 0,10 & 0,21 & $0^{*}$ \\
\hline$L C$ & 0,36 & 0,37 & 0,47 & $4,5^{\star}$ \\
\hline \multicolumn{5}{|l|}{ Groupe B } \\
\hline$d l$ & 290 & 286 & 286 & \\
\hline$P V$ & 9,6 & 13,4 & 8,2 & 0,01 * \\
\hline$H T$ & 11,48 & 10,07 & 11,44 & 45,6 NS \\
\hline$D M$ & $1,062.10^{-3}$ & $0,666.10^{-3}$ & $1,171.10^{-3}$ & $0^{*}$ \\
\hline$L F$ & 0,13 & 0,13 & 0,21 & $0^{*}$ \\
\hline$\angle C$ & 0,37 & 0,38 & 0,44 & $28,1 \mathrm{NS}$ \\
\hline
\end{tabular}

$d l$ : degrés de liberté; probabilité : probabilité $\alpha$ calculée pour le test de Bartlett. * : significatif, NS : non significatif. PV, HT, DM, LF, $L C$ : caractères étudiés (cf texte).

L'évolution de $\sigma^{2}$, en fonction du coefficient $F$, est la même dans les 2 groupes pour un caractère donné, sauf pour le caractère HT. Mais il n'y a pas de comportement général pour cette évolution, si l'on considère les cinq caractères; par exemple $\sigma^{2}$, s'élève entre les populations $A F_{1}$ et $\mathrm{AF}_{2}$ dans les 2 groupes pour les caractères $D M$, $L F, L C$ ou décroît pour le caractère $P V$.

\section{Variances interfamilles $\sigma 2_{F}$ (tableau II)}

Certaines variances $\sigma^{2} F$ n'étaient pas significativement différentes de 0 selon un test de $F$; particulièrement celles de la génération SIB dans le groupe B, pour tous les caractères. Certaines variances statistiquement non différentes de 0 dans une population ne l'étaient pas dans une autre

Tableau II. Variances interfamilles $\sigma_{F}^{2}$ : valeurs et comparaisons par un test de Bartlett pour les 3 populations.

\begin{tabular}{|c|c|c|c|c|}
\hline Variable concernée & $S I B$ & $A F_{1}$ & $A F_{2}$ & Probabilité \\
\hline \multicolumn{5}{|l|}{ Groupe A } \\
\hline$d l$ & 4 & 4 & 4 & \\
\hline$P V$ & $3,70 * 1$ & $0,60 \mathrm{NS}^{1}$ & $1,23 * 1$ & $96 \mathrm{NS}^{2}$ \\
\hline$H T$ & $7,5 *$ & $8,8^{*}$ & $7,5^{\star}$ & 77,4 NS \\
\hline$D M$ & $0,201 \cdot 10^{-3}$ * & $0,124.10^{-3}$ * & $0,089 \cdot 10^{-3} \mathrm{NS}$ & $84,1 \mathrm{NS}$ \\
\hline$L F$ & $0,07^{*}$ & $0,03 *$ & $0,01 \mathrm{NS}$ & 80,9 NS \\
\hline$L C$ & $0,01 \mathrm{NS}$ & $0,051^{\star}$ & 0,09 * & 53,8 NS \\
\hline \multicolumn{5}{|l|}{ Groupe B } \\
\hline$d l$ & 4 & 4 & 4 & \\
\hline$P V$ & $0,68 \mathrm{NS}$ & 10,20 * & $5,12^{*}$ & 14,8 NS \\
\hline$H T$ & ONS & $6,30^{*}$ & $11,79^{*}$ & $3,7^{*}$ \\
\hline$D M$ & $0,003 \cdot 10^{-3} \mathrm{NS}$ & $0,238 \cdot 10^{-3}$ * & $0,378 \cdot 10^{-3}$ * & $17,6 \mathrm{NS}$ \\
\hline$L F$ & 0,008 NS & $0,01 \mathrm{NS}$ & 0,007 & 76,9 NS \\
\hline$L C$ & $0,02 \mathrm{NS}$ & 0,02 * & $0,13^{*}$ & $2,0^{*}$ \\
\hline
\end{tabular}

$\mathrm{dl}$ : degrés de liberté; ${ }^{1}$ : variance $\sigma_{F}^{2}$ statistiquement différente de zéro, NS variance $\sigma_{F}^{2}$ statistiquement non différente de $0 .{ }^{2}$ : signification du test de Bartlett, ${ }^{\star}$ : significatif, NS : non significatif. 
( $D M, P V, L C$ dans le groupe $A$, tous les caractères excepté $P V$ dans le groupe $B$ ).

Les estimations des variances interfamilles ne diffèrent pas entre les populations, excepté pour les caractères $H T$ et $L C$ dans le groupe $B$.

Dans chaque groupe, la consanguinité influence les variances intrafamilles mais n'affecte pas les variances interfamilles.

\section{Comparaisons des variances des 2 groupes dans une population donnée}

\section{Variances intrafamille $\sigma^{2}$, (tableau III)}

Le nombre de degrés de liberté étant élevé, les résultats des tests sont fiables. Les différences ne sont pas significatives sauf pour $L F$ dans la population $A F 1$ et $P V$ pour la population $\mathrm{AF}_{2}(F$ calculé égal à la valeur au seuil $5 \%$ ). La présence du gène Rm1 ne semble pas influencer la variance intrafamille.

\section{Variances interfamilles $\sigma_{F}^{2}$ (tableau IV)}

La seule différence significative concerne le caractère $H T$ à la génération SIB, la variance du groupe $B$ étant très faible. Le gène $R m 1$ n'affecte pas les variances interfamilles.

\section{DISCUSSION}

L'évolution des variances $\sigma^{2}$, ne pouvait être aisément prédite. En général, les variances génétiques intrafamilles décroissent lorsque la consanguinité augmente (Hallauer et Miranda, 1981). Mais parallèlement, les variances environnemen-

Tableau III. Comparaison des variances intrafamilles $\sigma^{2}$, des groupes $A$ et $B$ par un test de $F$.

\begin{tabular}{llll}
\hline $\begin{array}{l}\text { Variable } \\
\text { concernée }\end{array}$ & SIB & \multicolumn{1}{c}{$A F_{1}$} & $A F_{2}$ \\
\hline & & & \\
$d l$ & $(294290)$ & $(294290)$ & $(273286)$ \\
$P V$ & $1,20 \mathrm{NS}$ & $1,04 \mathrm{NS}$ & 1,24 \\
$H T$ & $1,03 \mathrm{NS}$ & $1,21 \mathrm{NS}$ & $1,20 \mathrm{NS}$ \\
$D M$ & $1,07 \mathrm{NS}$ & $1,02 \mathrm{NS}$ & $1,17 \mathrm{NS}$ \\
$L F$ & $1,10 \mathrm{NS}$ & $1,30^{*}$ & $1,00 \mathrm{NS}$ \\
$L C$ & $1,03 \mathrm{NS}$ & $1,03 \mathrm{NS}$ & $1,07 \mathrm{NS}$ \\
& & & \\
\hline
\end{tabular}

$F 5 \%=1,24 ;{ }^{*}$ significatif, NS non significatif.
Tableau IV. Comparaisons des variances interfamilles des groupes $A$ et $B$ par un test de $F$.

\begin{tabular}{llll}
\hline $\begin{array}{l}\text { Variable } \\
\text { concernee }\end{array}$ & \multicolumn{1}{c}{$S I B$} & $A F_{1}$ & $A F_{2}$ \\
\hline & & & \\
\hline$P V$ & $3,54 \mathrm{NS}$ & $9,35 \mathrm{NS}$ & $3,66 \mathrm{NS}$ \\
$H T$ & $16,0^{*}$ & $1,45 \mathrm{NS}$ & $1,52 \mathrm{NS}$ \\
$D M$ & $3,86 \mathrm{NS}$ & $1,82 \mathrm{NS}$ & $2,86 \mathrm{NS}$ \\
$L F$ & $8,40 \mathrm{NS}$ & $2,00 \mathrm{NS}$ & $1,00 \mathrm{NS}$ \\
$L C$ & $2,86 \mathrm{NS}$ & $3,00 \mathrm{NS}$ & $1,40 \mathrm{NS}$ \\
\hline
\end{tabular}

$F 5 \%, 4,4=9.6$.

tales tendent plutôt à augmenter du fait d'une plus grande sensibilité des génotypes à l'environnement. Chez Petunia hybrida, l'effet de l'environnement sur le phénotype peut être très important (Brunaud et al, 1977) et nous avons vérifié que les variances des lignées (génotypes homozygotes) sont plus grandes que celles des hybrides $F_{1}$ (génotypes hétérozygotes), la différence étant plus ou moins prononcée selon le caractère considéré (Robert, 1989). Si l'effet de la consanguinité sur la variance environnementale est faible (ce qui est le cas des caractères $D M$ et $L F$ qui présentent des écarts entre variances d'environnement des lignées et des hybrides moins importants que les caractères $P V$ et $H T$, les variations de la variance intrafamille entre les générations $A F_{1}$ et $A F_{2}$ dépendent principalement des variations de la composante génétique de cette variance. La variance génétique de chaque famille dépend du niveau de consanguinité de la plante choisie pour l'obtenir. Le coefficient de consanguinité calculé pour une population en ségrégation n'est qu'un coefficient moyen : les coefficients individuels sont variables. L'effectif de plantes retenues pour représenter chaque population étant faible (5), les effets d'échantillonnage pour le niveau de consanguinité des plantes choisies sont importants. En conséquence, la valeur moyenne de la variance intrafamille de leurs descendants en autofécondation peut être notablement plus élevée (cas observé des caractères $D M$ et $L F$ ) ou plus faible que celle qui serait attendue. Les comparaisons entre générations faites famille par famille pour chaque groupe (données non publiées) montrent des évolutions très variables pour un même caractère, ce qui semble un autre élément indiquant le caractère aléatoire de ces évolutions. 
La puissance des tests $F$ est probablement en cause pour le nombre élevé de variances $\sigma_{F}^{2}$ statistiquement nulles. Avec 5 familles étudiées par population, la probabilité $\beta$ d'accepter l'hypothèse nulle quand elle est fausse est élevée (Steel et Torrie, 1960). Du fait des effets d'échantillonnage, la variabilité présente dans nos échantillons est probablement très différente de la variabilité des populations $\mathrm{SIB}, A F_{1}$ et $A F_{2}$. La population SIB du groupe $B$ présente le nombre le plus élevé de variances $\sigma_{F}^{2}$ statistiquement nulles. Cela pourrait expliquer les différences observées entre les variances des 3 populations dans le groupe $B$ (caractères $H T$ et $L C$ ). Les variances obtenues par autofécondation n'étant pas nulles, les données suggèrent que l'intercroisement ait pu conduire à la formation de variabilité potentielle, telle que définie par Mather (1943).

Dans cette expérience, le gène Rm1 qui accroît les taux de recombinaison dans la plupart des segments testés, excepté le segment sur le chromosome IV délimité par les gènes B1 et An6 (Farcy et al, 1986; Cornu et al, 1989; Robert et al, 1991), n'a pas d'influence sur la variance des caractères quantitatifs étudiés. Plusieurs hypothèses génétiques peuvent être émises :

- les gènes contrôlant les 5 caractères sont liés mais ne réagissent pas à l'action de Rm1,

- les gènes peu nombreux sont indépendants ou présentent des liaisons faibles, si bien que les 2 groupes ne pouvaient être considérés comme différents pour la recombinaison.

Ces 2 hypothèses supposent un déterminisme des caractères peu adapté à l'étude entreprise. Cependant, des causes statistiques peuvent aussi être invoquées : le petit nombre de familles (5) dans chaque groupe impliquait que les différences entre groupes devaient être particulièrement élevées pour être déclarées significatives.

Enfin, l'hypothèse que les réarrangements chromosomiques occasionnés par la recombinaison méiotique ne se soient pas répercutés sur la variance génétique ne peut être négligée. Pour un caractère purement additif, la recombinaison a des effets opposés sur la variance génétique, suivant que les loci sont initialement en couplage ou en répulsion (Miller et Rawling, 1967; Mather et Jinks, 1971; Gallais, 1974, Snape, 1976; Snape et Simpson, 1981). L'importance relative des associations en couplage et répulsion peut donc modifier l'impact de la recombinaison sur la valeur de la variance génétique; c'est une autre cause possible de l'absence de différence constatée entre les 2 groupes de plantes.

La nécessité de pratiquer des croisements de contrôle pour déterminer le génotype au locus Rm1 (générations supplémentaires, nombre de descendances testées élevé) n'a pas permis de travailler avec un effectif de familles par population SIB, $A F_{1}, A F_{2}$, suffisant, ce qui entache d'incertitude nos résultats. Aussi avons-nous pratiqué des simulations par micro-ordinateur afin de lever les contraintes expérimentales et les contraintes génétiques évoquées ci-dessus, qui peuvent être à l'origine des résultats expérimentaux obtenus (Kervella et al, 1993).

\section{RÉFÉRENCES}

Brunaud A, Bugnon F, Cornu A (1977) Analyse du port chez des pétunias. Bull Soc Bot Fr 124, 307327

Cornu A, Farcy E, Mousset C (1989) A genetic basis for variation in meiotic recombination in Petunia hybrida. Genome 32, 46-53

Cornu A, Farcy E, Maizonnier D, Haring M, Weerman W, Gerats AGM (1990) Petunia hybrida. In: Genetic maps (SJ O'Brien, ed) Cold Spring Harbor Lab Press, 5th edn, 107-124

Farcy E, Mousset C, Maizonnier D, Cornu A (1986) Genetic regulation of meiotic recombination in Petunia hybrida. In: Symp int eucarpia, genetic manipulation in plant breeding, 145-147

Gallais A (1974) Covariances between arbitrary relatives with linkage and epistasis in the case of linkage disequilibrium. Biometrics 30, 429-446

Gallais A (1981) Amélioration des populations en vue de la création de variétés. Sélectionneur Fr 29, 5-23

Hallauer AR, Miranda JB (1981) Quantitative genetics in maize breeding. lowa State Univ, Ames, IA, $468 \mathrm{p}$

Kervella J, Robert N, Fouilloux G (1993) Influence de la recombinaison sur la variabilité génétique. II. Étude par simulations. agronomie (sous presse)

Mather K (1941) Variation and selection of polygenic characters. J Genet 41, 159-193

Mather K (1942) The balance of polygenic combinations. J Genet 43, 309-336

Mather K (1943) Polygenic inheritage and natural selection. Biol Rev 18, 32-64

Mather K, Jinks JL (1977) Introduction to biometrical genetics. Cornell Univ Press, Ithaca, New York

Miller PA, Rawling JO (1967) Breakup of initial blocks through intermating in cotton breeding populations. Crop Sci 7, 199-204

Mousset C (1985) Etude des facteurs génétiques contrôlant la fréquence des taux de recombinaison 
entre chromosomes homologues chez Petunia hybrida Hort. Thèse de l'université de Rennes I, $193 p$

Robert N (1989) Etude d'un gène modificateur des taux de recombinaison chez Petunia hybrida et de son incidence sur la variabilité de caractères quantitatifs. Thèse de l'université de Rennes I, France

Robert N, Farcy E, Cornu A (1991) Genetic control of meiotic recombination in $P$ hybrida: dosage effect of gene $\mathrm{Rm} 1$ on segments $\mathrm{Hf} 1-\mathrm{Lg} 1$ and An2-Rt; role of modifiers. Genome 34, 515-523

Searle SR (1971) Topics in variance components estimation. Biometrics 27, 1-76
Snape JW (1976) A theoretical comparison of diploised haploid and single seed descent population. Heredity 36, 275-277

Snape JW, Simpson E (1981) The genetical expectation of doubled haploid lines derived from different filial generations. Theor Appl Genet 60, 123-133

Spiess EB, Allen AC (1961) Release of genetic variability through recombination. VI Second and third chromosomes of Drosophila melanogaster. Genetics 46, 1531-1553

Stell RGG, Torrie JH (1960) Principles and procedures of statistics. Mc Graw Hill Book Co, 2nd edn, $418 \mathrm{p}$ 